\title{
Violencia obstétrica: haciendo visible lo invisible
}

\section{Obstetric violence: making the invisible visible}

\author{
Elisa Jojoa-Tobar'; Yuler-Darío Cuchumbe-Sánchez ${ }^{1}$; Jennifer-Briyith Ledesma-Rengifo'; \\ María-Cristina Muñoz-Mosquera ${ }^{1}$; Adriana-María Paja-Campo ${ }^{1}$; Juan-Pablo Suarez-Bravo ${ }^{1}$
}

Forma de citar: Jojoa-Tobar E, Chuchumbe-Sánchez YD, Ledesma-Rengifo JB, Muñoz-Mosquera MC, Paja-Campo AM, Suárez-Bravo JP. Violencia obstétrica: haciendo visible lo invisible. Rev Univ Ind Santander Salud. 2019; 51(2): 135-146. doi: http://dx.doi.org/10.18273/revsal.v51n2-2019006 (c) (1)

\section{Resumen}

Introducción: La violencia obstétrica ha sido tipificada como una modalidad de violencia de género, que hace referencia a las conductas de acción u omisión; puede ser institucional, psicologica, simbólica o sexual. Objetivo: Caracterizar las diferentes modalidades de violencia obstétrica. Metodología: Estudio cualitativo con enfoque fenomenológico; conformado por 16 mujeres con experiencias obstétricas menores de dos años que asistieron a los hogares de Familia, Mujer e Infancia (FAMI) del Instituto Colombiano de bienestar familiar en la ciudad de Popayán (Colombia) durante el 2016. Se realizaron entrevistas estructuradas y a profundidad sobre sus experiencias en la atención institucional del parto, en términos de toma de decisiones e información sobre procedimientos, posición para el parto, presencia de acompañante, derecho a la intimidad, percepciones sobre maltrato físico y psicológico. Resultados: El 69\% de las entrevistadas reportaron alguna forma de violencia obstétrica. La violencia institucional se registró en la mayoría de los relatos y estuvo asociada a políticas institucionales que no satisfacen las expectativas de las gestantes en el proceso de parto e inconformidad con la atención en salud recibida durante el trabajo de parto; la violencia psicológica, fue percibida como falta de información y de autonomía en la toma de decisiones en el proceso de atención, malos tratos e impedimento a las gestantes para expresar sus sentimientos y emociones. Conclusiones: La mayoría de mujeres desconocen sus derechos y/o naturalizan los actos de violencia obstétrica. Los profesionales de salud deben reconocer en su formación y en los campos de práctica, los distintos tipos de violencia obstétrica con el fin de diseñar mecanismos para su prevención y abolición. Se hace necesario explorar la ocurrencia de eventos asociados a violencia sexual en el marco de la atención obstétrica.

Palabras clave: Violencia obstétrica; Parto; Derechos sexuales y reproductivos.

\begin{abstract}
Introduction: Obstetric violence has been typified as a form of gender violence, which refers to the behaviors of action or omission; It can be institutional, psychological, symbolic or sexual. Objective: To characterize the different modalities of obstetric violence. Methodology: Qualitative study with a phenomenological approach; conformed by 16 women with obstetric experiences under two years who attended the homes of Family, Women and Children (FAMI) of the Colombian Institute of Family Welfare in the city from Popayán (Colombia) during the 2016.

1. Universidad del Cauca. Popayán, Colombia

Correspondencia: Elisa Jojoa Tobar. Dirección: Cra. 5 13N-36 Popayán. Teléfono:+573108354263. Correo electrónico: elisaj@unicauca. edu.co
\end{abstract}


Structured interviews were conducted and in depth about their experiences in the institutional care of childbirth, in terms of decision making and information about procedures, position for childbirth, presence of companion, right to privacy, perceptions about physical and psychological abuse. Results: $69 \%$ of the interviewed women reported some form of obstetric violence. Institutional violence was recorded in most of the reports and was associated with institutional policies that do not meet the expectations of pregnant women in the birth process and nonconformity with health care received during labor; Psychological violence was perceived as a lack of information and autonomy in decision-making in the process of care, mistreatment and impediment to pregnant women to express their feelings and emotions. Conclusions: The majority of women are unaware of their rights and / or naturalize acts of obstetric violence. Health professionals must recognize in their training and in the fields of practice, the different types of obstetric violence in order to design mechanisms for their prevention and abolition. It is necessary to explore the occurrence of events associated with sexual violence within the framework of obstetric care.

Keywords: Obstetric violence; Birth; Sexual and reproductive rights.

\section{Introducción}

Garantizar el acceso universal a una salud sexual y reproductiva segura, aceptable y de buena calidad, especialmente a la atención de la salud en el embarazo, puede reducir drásticamente los índices globales de morbilidad y mortalidad materna y perinatal; sin embargo, los avances logrados en tecnología, cobertura de atención, infraestructura, cualificación del personal asistencial e incremento sostenido de la cobertura institucional del parto han ido en detrimento de la posibilidad de que las mujeres transiten por la maternidad como una experiencia natural, gozosa y saludable para su pareja y su familia, convirtiéndose en un hecho biológico, patologizado, institucionalizado y despersonalizado, en el cual la mujer pierde la autonomía sobre su cuerpo.

En estos procesos de atención emergen diferentes manifestaciones del modelo patriarcal y del modelo médico hegemónico, representados en un mayor interés en la productividad, en la disminución de los costos de atención, en una medicina basada en la evidencia que desconoce que uno de sus pilares es la interlocución con el paciente sobre sus procesos de atención, donde las relaciones asimétricas entre las maternas y el personal de salud, son el común denominador y el "sometimiento" al saber médico y a las normas del sistema de salud, las cuales desdibujan la autonomía de la mujer para decidir sobre como asumir el proceso de trabajo de parto y el parto.

En consecuencia, en la violencia obstétrica convergen la violencia institucional en salud y la violencia de género. Por una parte, la violencia institucional es definida por Bruyn $^{1}$ como "una forma específica de abuso, que comprende daño físico y psicológico a personas como resultado de condiciones estructuralmente inadecuadas en las instituciones y sistemas públicos" considerando además que está estrechamente relacionada con el trato degradante y la baja calidad de la atención en salud. Por otra parte, la violencia de género ha sido definida por el Ministerio de Salud y Protección Social ${ }^{2}$ como "cualquier acción o conducta que se desarrolle a partir las relaciones de poder asimétricas basadas en el género que sobrevaloran lo relacionado con lo masculino y subvaloran lo relacionado con lo femenino".

Es así, como la violencia obstétrica ha sido tipificada como una modalidad de violencia de género, que hace referencia a las conductas de acción u omisión que se derivan del proceso de atención del embarazo, trabajo de parto, parto y puerperio en el ambiente hospitalario que incluyen: el maltrato físico y verbal, la humillación, los procedimientos médicos sin consentimiento o coercitivos (incluida la esterilización), la falta de confidencialidad, el incumplimiento con la obtención del consentimiento informado, la negativa a administrar analgésicos, las violaciones flagrantes de la privacidad, el rechazo de la admisión en centros de salud y la retención de las mujeres y de los recién nacidos debido a su incapacidad de pago ${ }^{3}$.

Además de estos componentes que vulneran los derechos de la mujer, la Organización Mundial de la Salud (OMS), ha hecho un llamado frente al incremento injustificado de la cesárea a nivel mundial; es así como en Latinoamérica casi cuatro de cada diez partos son por este método $(38,9 \%)$, cifra muy por encima de lo recomendado por esta organización (10\% y 15\%) desde hace 40 años

Aunque estudios afirman ${ }^{5-7}$ que la violencia obstétrica se ejerce frecuentemente, existen dificultades reales para que las mujeres denuncien; para empezar, entre las pacientes no hay conciencia de sus derechos durante la atención del embarazo, parto, postparto o post-aborto; tampoco esta problemática se difunde de manera 
sostenida; finalmente, la violencia obstétrica está tan naturalizada entre las mujeres que la viven, que resulta difícil conceptualizarla como tal.

En el contexto latinoamericano se destacan los avances normativos en México, Argentina y Venezuela; en Colombia no hay una legislación que prevenga y sancione este tipo de violencia, por lo tanto, ha sido invisibilizada y a nivel investigativo apenas comienza a recolectarse evidencia que permita abordar esta problemática. Más allá de las implicaciones que tiene en la calidad de la prestación de servicios de salud, se hace necesario posicionarla como un tema de defensa de los derechos humanos de las mujeres que requiere ser intervenido mediante la construcción de un marco legislativo, la modificación de guías de práctica clínica, protocolos de atención e inclusión dentro de los diseños curriculares de la formación del personal de salud y el empoderamiento de las mujeres de sus procesos sexuales y reproductivos en el marco de la prestación de los servicios de salud.

Con el fin de visibilizar esta problemática en el contexto local y proponer acciones en los contextos institucionales, territoriales y comunitarios, se propuso como objetivo caracterizar las diferentes modalidades de violencia obstétrica en mujeres del programa "Hogares FAMI" con experiencia obstétrica reciente.

\section{Materiales y métodos}

Para llevar a cabo esta investigación se realizó un estudio cualitativo con enfoque fenomenológico. La población estuvo conformada por 16 madres de niños menores de dos años que asistieron a los hogares de la Asociación 31 de Marzo pertenecientes al programa FAMI del ICBF durante el I semestre del 2016; considerando que al tener una experiencia obstétrica reciente, la información obtenida sería mucho más fidedigna y cargada de más detalles que serían relevantes para la investigación. Por otra parte, los hogares FAMI tienen dentro de su población sujeto, mujeres gestantes y lactantes con niños hasta de dos años, lo que posibilitó el encuentro con esta población.

Las técnicas de recolección utilizadas incluyeron una entrevista estructurada, para la identificación de los aspectos sociodemográficos y una entrevista a profundidad para la recolección de las experiencias vividas en el proceso de trabajo de parto, parto y puerperio y la obtención de datos relacionados con las modalidades de violencia obstétrica percibida en la entidad de salud.
Las entrevistas se realizaron durante las jornadas de encuentro programadas por las madres FAMI. Se socializó en cada encuentro los propósitos de la investigación y se explicaron los términos del consentimiento informado para ser firmado a continuación por cada una de las participantes. Los investigadores registraron las entrevistas por medio de grabaciones de audio, posteriormente fueron trascritas en su totalidad en formato Excel, archivadas con un código asignado para su identificación y analizadas identificando las categorías y subcategorías. Cada investigador registró los datos sociodemográficos de la participante y con base en una guía de entrevista a profundidad exploró la experiencia de la mujer en relación con su experiencia personal durante la atención de su parto en el contexto institucional.

Esta investigación se basó en los principios estipulados en CIOMS/82 -2002 y en la resolución 008430 de 4 de octubre de 1993, en la que se establece las conductas científicas, técnicas y administrativas para la investigación en salud. De igual manera, estuvo regida a los principios de autonomía, beneficencia, no maleficencia, justicia, objetividad y confidencialidad.

\section{Resultados y discusión}

Un $12 \%$ de las participantes tenían entre 15 a 19 años, $57 \%$ de 20 a $25,19 \%$ de 26 a 35 y $12 \%$ eran mayores de 36 años. Respecto al nivel educativo, el $31 \%$ cursó el bachillerato; el mismo porcentaje afirmó no haber culminado la primaría. De la información sociodemográfica, es de resaltar que el $63 \%$ de las informantes refirió tener un hijo; con relación a la ocupación, la mayoría manifestó ser ama de casa.

De las 23 entrevistas realizadas en el $69,5 \%$ se evidenció una o más formas de violencia obstétrica, lo que implica que este evento resulta ser muy frecuente y evidencia la vulneración de los derechos humanos de las mujeres, así como de sus derechos sexuales y reproductivos.

El aspecto central de la investigación parte de la comprensión del concepto de la violencia obstétrica como el conjunto de prácticas, comportamientos y actitudes que afectan a la gestante de modo negativo en el desarrollo de su trabajo de parto, parto y puerperio. Las diferentes modalidades de violencia obstétrica fueron sistematizadas en un mapa con las categorías y subcategorías consideradas para el estudio tal como lo muestra la siguiente figura. 


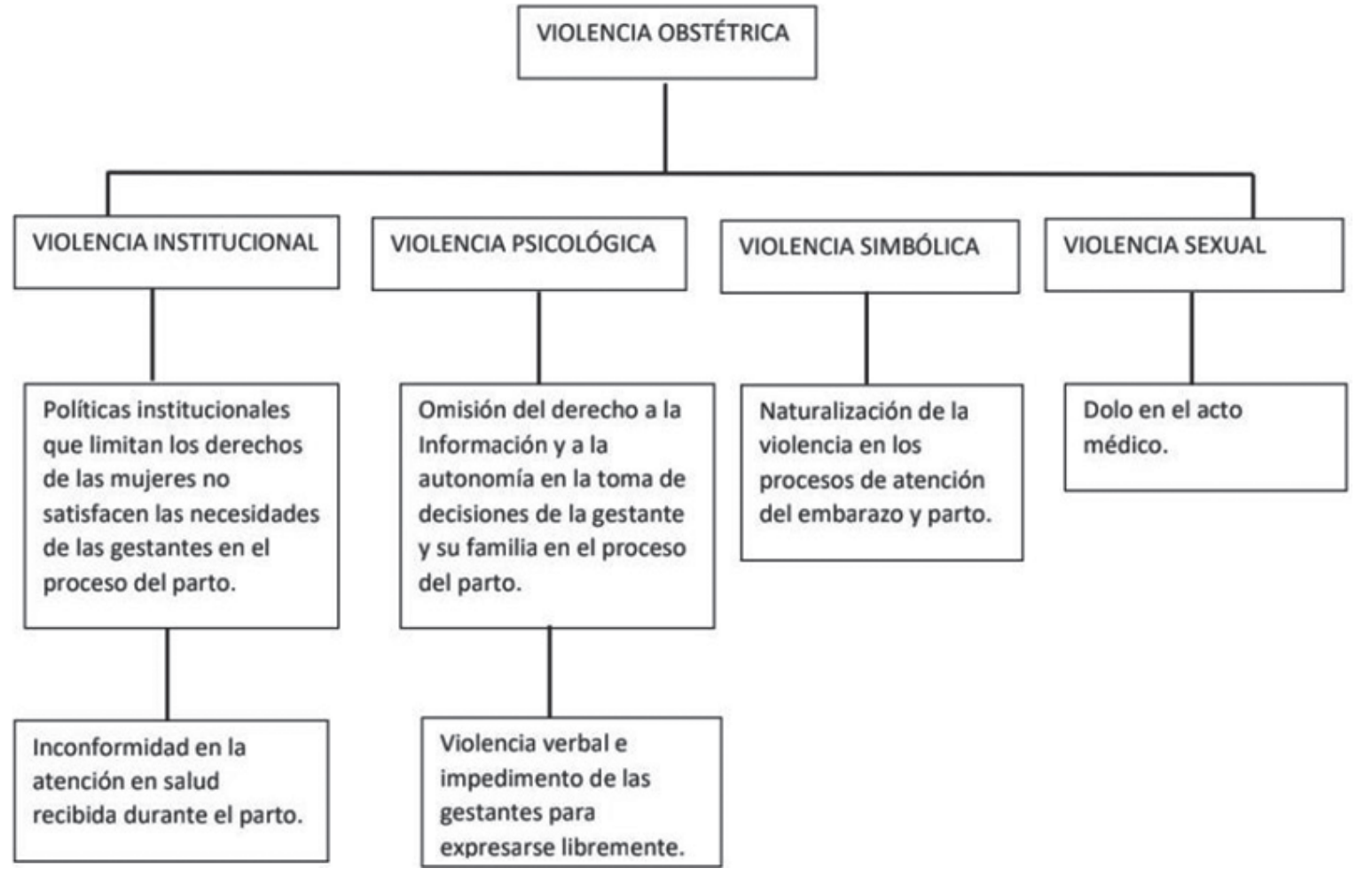

FIGURA 1. Mapa de relación de categorías con subcategorías Fuente: Elaboración propia.

\section{Violencia institucional}

Se manifiesta según Bruyn ${ }^{1}$ como: negar tratamiento sin referir a otros servicios para recibir asistencia oportuna, proveer deliberadamente información defectuosa o incompleta, largas esperas para ser atendida en las instalaciones de salud que pueden contribuir a aflicciones psicológicas y daños físicos, negación de medicamentos, atención o manejo del dolor como 'castigo' y tarifas excesivas por los servicios.

Esta modalidad de violencia obstétrica fue subcategorizada en: políticas institucionales que no satisfacen las expectativas de las gestantes en el proceso de parto e inconformidad con la atención en salud recibida durante el trabajo de parto. Cabe resaltar que la violencia institucional fue la mayormente referenciada en los relatos.

Las políticas institucionales son decisiones escritas que se establecen como manuales guías o protocolos de atención, en relación a los procedimientos administrativos y de prestación de los servicios de salud. Estas juegan un papel importante en la garantía de los derechos de las mujeres durante la atención en el proceso obstétrico, contribuyendo a un servicio humanizado y de calidad. Sin embargo, los rígidos marcos institucionales y el desconocimiento de las prácticas de cuidado cultural, vulneran con frecuencia la posibilidad de vivir el parto como una vivencia positiva, integral y en familia.

De esta manera, en el estudio se hallaron algunas políticas institucionales relacionadas con el ejercicio docente que generan inconformidades, al no estar reguladas o no ser explícitamente aprobadas por las gestantes. Principalmente, se refirieron al tacto para evaluar la dilatación, puesto que es realizado de manera repetitiva, por distintas personas y $\sin$ un consentimiento, Quevedo ${ }^{8}$ evidencia en estos actos médicos la vulneración del derecho a la intimidad por la intromisión no consentida de la privacidad mediante la exhibición y/o revisión masiva del cuerpo y los órganos genitales.

Todas estas situaciones generan en las mujeres ansiedad, sensación de indefensión e incomodidad: "Me realizaron muchos tactos el sábado y el domingo. Me sentía incómoda. A cada rato tactos del médico y un pasante. El médico sacaba la mano y luego hacia el tacto el pasante. - Y mire es así-... -Vea así es ... jaqui está! y imire! - La verdad muy incómodo." (Informante cultural 2). 
Según Vallana ${ }^{9}$ los tactos vaginales a repetición hacen parte de la "cadena de intervenciones" que apuran el parto en el modelo industrializado de parir/nacer" y que buscan que el proceso de nacer sea rápido y ocupe el menor tiempo posible del personal de salud. De tal manera que casi sistemáticamente se incluye un espiral de intervenciones en las cuales el parto debe ser inducido si este no avanza solo y prontamente. Con los tactos vaginales se expone también a la mujer a adquirir infecciones por la introducción de bacterias de la vagina en el útero.

El "someterse" a la atención de un parto institucional da por sentado en muchos casos la omisión por parte del personal de salud del consentimiento informado aún para procedimientos que desde el proceso de atención se consideran rutinarios: "Muchos tactos vaginales y además que entraban los estudiantes sin pedirme permiso, si yo quería que ellos estuvieran ahi, simplemente entraban y miraban." (Informante cultural 10). Al respecto Vallana ${ }^{9}$ señala que el miedo de las parturientas, encontrándose en un lugar extraño, frío, impersonal y bajo la mirada y manipulación de extraños, es por sí mismo un impedimento para el "normal" desarrollo del parto.

El miedo y la sensación de soledad son vividas por las mujeres desde el mismo momento en que llegan al hospital y son informadas que su pareja o familiar no la pueden acompañar ni en el proceso de trabajo de parto ni en el parto. Resulta contradictorio que, aunque la guía de práctica clínica resultado de medicina basada en la evidencia recomienda que la mujer en trabajo de parto sea acompañada de manera individual y de forma continua por la persona que ella elija ${ }^{10}$, el común denominador en las instituciones de salud es prohibir la participación del padre o de un familiar en este proceso de atención. "Yo pensé que iba a dejar al papá de mi bebé o a mi hermana (pensaba que el personal de salud iba a autorizar el ingreso de los familiares para el acompañamiento continuo a la madre en el trabajo de parto y parto)... pero no, no me dejaron (el personal de salud) ni tener un teléfono ni nada y no dejaron pasar a mi mamá que estaba en la sala de espera, y yo sola." (Informante cultural 8).

Según Arguedas ${ }^{11}$, al compañero de la mujer embarazada se le asigna la tarea de esperar en silencio, pues es considerado como un estorbo en el proceso de parto, mientras que para la gestante su presencia es indispensable, es apoyo, es solidaridad, es el transitar juntos por la experiencia de convertirse en padres “(...) uno necesita más ayuda y ese apoyo y uno estar como solo allá, eso allá no dejan, que siempre los familiares tienen que esperar afuera (...) Eso sí me gustaría que cambiara, que permitieran que el papá estuviera ahí presente."(Informante cultural 11).

Resulta paradójico que la concepción sea una vivencia de pareja pero que la llegada del fruto de esa concepción sea una experiencia vivida en soledad. Existe evidencia ${ }^{12}$ de que la participación del padre en el proceso de trabajo de parto y parto contribuye a una mayor participación del padre en las labores de crianza del hijo, en el fortalecimiento del vínculo padre-hijo y en el mejoramiento de la relación de pareja entre otros aspectos.

En Chile la participación del padre en los procesos de atención hace parte de las metas institucionales a cumplir lo que ha permitido un incremento del 18 al $55 \%$ del acompañamiento de los partos por la pareja o un familiar cercano. ${ }^{13}$ De allí que las políticas institucionales son determinantes para garantizar un efectivo acompañamiento a la gestante, lo que impactaría positivamente en la disminución de este tipo de violencia considerando que la presencia de un familiar o persona significativa evitaría la condición de indefensión total de la parturienta que se encuentra en situación de vulnerabilidad. Así mismo, significaría un gran apoyo para el personal de salud que contaría con personas que acompañen procesos como la deambulación segura en el trabajo de parto, suministro de líquidos por vía oral, realización de masajes, entre otras y la contención emocional que es fundamental en esta etapa; para ello se requiere sin embargo la orientación por parte del personal de salud.

Las políticas institucionales inciden negativamente sobre la autonomía de la mujer para decidir sobre la posición para su trabajo de parto y parto. "No estaba cómoda, si solamente acostada, intente pararme y estar como de rodillas porque asi el dolor era como menos (dolor de las contracciones), pero me regañaron (el personal de salud), porque de acuerdo a eso él bebe se me podía venir (acelerar el proceso de parto), y se me podía golpear, pero pues me dijeron que no (personal de salud) y si me regañaron varias veces." (Informante cultural 8).

Al respecto, Vallana9 señala que "aparte de la inmovilización propiciada por los artilugios tecnológicos, las parturientas deben ser protegidas de golpes y caídas dentro de las instituciones hospitalarias, lo que hace que se les prohíba deambular, caminar, agacharse, acurrucarse, ponerse en cuatro, entre otros, 
movimientos todos estos que contribuyen a la dilatación y ayudan a hacer las contracciones más llevaderas. Así, la imposición de la quietud es entonces inadecuada para la fisiología del parto, así como también la posición supina". Sin duda la adopción de una postura supina para el trabajo de parto y parto hace parte del proceso de "sometimiento" a la atención institucional del parto, lo que se traduce en impotencia y falta de contención para el dolor que la mujer experimenta en dicha posición, pues le resulta más dolorosa y retrasa lo que justamente se busca por parte del personal de salud: "que el parto sea rapidito". Las nuevas recomendaciones de la OMS ${ }^{14}$ que buscan hacer del parto una experiencia positiva respaldan la libre determinación de la mujer para tener su parto en la posición que les resulte más cómoda, lo que disminuiría el tiempo del parto, el dolor y la inconformidad de las mujeres durante los procesos de atención entre otros beneficios.

Todas estas situaciones permitieron identificar otra subcategoría denominada "Inconformidad en la atención en salud recibida durante el trabajo de parto", que las mujeres perciben al encontrar que sus solicitudes, manifestaciones de dolor, incomodidad y necesidad de compañía son ignoradas, por la falta de contención y burlas principalmente cuando las mujeres vivencian por primera vez este proceso: "Me gustaría una atención mejor tanto para uno como para las demás, estar más pendientes ellos (personal de salud) deberían decirle a uno no es tiempo. La dejaban mucho tiempo sola. La oían quejar (gestante en trabajo de parto que compartía sala con otra) y lo que hacian era burlase por allá y decir que jesta muchacha si se queja!, ique si llora!, ique no es tiempo! (Informante cultural No. 5).

Las adolescentes y primerizas son las mujeres con mayor riesgo de ser víctimas de este tipo de violencia: " ¿Y uno irse a quejar? ¡No! Si eso la que se queja la empieza a regañar. Había una muchacha parecía primeriza, se quejaba muchísimo y ella gritaba.- jayy Dios! Estos dolores... ipor favor! (A los doctores les daba risa y decían -jay no mija!, ahí sí Dios, pero cuando se ponen a abrir las piernas ahí si no es Dios mío. Sólo: iqué rico! y vea acá es lo bueno. (Informante cultural 5).

El "castigo" por quejarse o gritar de dolor es el abandono, el dejarlas solas, el ignorar; táctica frecuente en los relatos obtenidos y que se constituyen en un hecho aleccionador para las demás mujeres con quienes se comparte estos espacios.

Villanueva - $\operatorname{Egan}^{15}$ señala que el disentimiento de las instrucciones médicas provoca una reacción en el personal de salud que puede llegar a ser determinante, con miras a reprimir cualquier intento de lo que, desde su punto de vista, puede ser considerado una insubordinación, frente a lo cual se evidencian actos de rebeldía o solidaridad que emerge en mujeres con tal vez algún grado mayor de interlocución que abogan por aquellas que están siendo atropelladas, pero resultan rápidamente neutralizadas por el personal de salud: "Uno quería decir algo y... ; a ver mamita, calladita. No opine!" (Madre que compartía sala partos y abogaba por su compañera) (Informante cultural 2).

El silencio entonces resulta ser finalmente la estrategia adoptada por las mujeres para conseguir de alguna manera una mejor atención.

Otra mujer refiere: "Mi miedo a que mi hijo fuera a nacer muerto. Ya no lo sentía. No manifestaba nada porque le decian -jay mamá, deje la bulla! Entonces como que no te dejan hablar y si vas hablar por tu compañerita (gestante comparte sala partos) ;Ay mamita eso es de cada una!... y a uno le pasan los dolores y va hablar por la otra compañerita. Entonces, jay mamá, quietica ahi, no se mueva no hable! (Informante cultural 2).

En estos contextos la sororidad, que se deriva de la hermandad entre mujeres al percibirse como iguales que pueden aliarse, compartir y sobre todo cambiar su realidad debido a la percepción de diversas maneras de opresión ${ }^{16}$, surge como último recurso, entre gestantes que comparten esta misma experiencia; sin embargo, estos pequeños actos de insurrección, que son rápidamente acallados y la sensación de soledad vuelve a surgir.

La atención a las mujeres en estos contextos, debe ser integral y de calidad por parte de todo el personal de salud. De este modo, los funcionarios que las reciben, los encargados de facturar el ingreso, el personal médico que las valora, el personal de enfermería que cuida de ellas y los auxiliares de enfermería a cargo, tienen el deber de realizar las intervenciones necesarias de manera ágil y oportuna para garantizar el bienestar de la paciente, teniendo en cuenta el protocolo que cada institución adopta frente a este servicio, pero considerando las necesidades individuales de las mujeres.

Respecto a la calidad de la atención en salud durante estos procesos, Sampaio ${ }^{17}$, refiere que las expectativas del cuidado de las gestantes en el parto, giran en torno a necesidades afectivas como cariño, atención y comprensión, y que estas deben ser satisfechas por el profesional de salud o por un acompañante para garantizar un servicio oportuno, de alta de calidad y humano. 
Sin embargo, se evidenció que estas expectativas no se cumplieron al presentarse demoras innecesarias, descuido en su estadía, discriminación, trato denigrante, subordinación, entre otras situaciones:

"Se demoraron al recibirme o sea en vez de darle prioridad a las que ya estamos en trabajo de parto, se demoraron, eso me toco pararme para decir que ya yo habia roto fuente". (Informante cultural 9). Resulta contradictorio que aunque a nivel institucional se promueva una discriminación positiva para las gestantes, la realidad evidencia que en algunos casos esta no se aplica, situación que es percibida por las gestantes como una mala calidad en la atención.

"De un momento a otro cuando fui al baño oriné sangre, sali y revente fuente. Fui al hospital y el ginecólogo me revisó y me iba a mandar para la casa a pesar que estaba sangrando". (Informante cultural 2).

Davis-Floyd ${ }^{17}$, citado por Vallana ${ }^{9}$ señala que, durante el parto, el mecanismo de ignorar a las madres y priorizar los resultados de los exámenes y tecnologías médicas aplicadas al parto es particularmente evidente, ya que el personal pasa constantemente a tomar las lecturas de los monitores sin siquiera interactuar con la paciente, uno de los relatos da cuenta de estas vivencias: "Fue una ginecóloga y me dijo que me dejaran que lo tuviera allá, que para que me ponía hacer escándalo. Yo le decía: - "pero es que yo ya siento que ella va a nacer" $y$ ella me decía que "todavía no va nacer. Si usted está en seis de dilatación”. - “'No!, yo siento que ya va a nacer, quiero hacer del cuerpo"- le decía. Ella me decía: - "pues hágase ahi"- cuando vino un ginecólogo y me metió la mano dijo: “No!, esa niña ya va a nacer”. Inmediatamente me acostaron en una camilla y cuando iba entrando a sala de partos ella salió (la bebé)".

El sentirse ignoradas es una sensación que con frecuencia apareció en los relatos y por las cuales se considera que existió una mala calidad en la prestación de los servicios de salud, al percibirse como desatención.

\section{Violencia Psicológica}

Los resultados de la investigación arrojaron que esta es la segunda modalidad con mayor frecuencia de aparición en los relatos, la primera subcategoría considera la omisión del derecho a la información y a la autonomía en la toma de decisiones de la gestante y su familia en el proceso del parto.
Cada parto es único, incluso para las mujeres no primerizas, por lo cual es necesario que la gestante esté informada sobre las decisiones que se tomen en el marco de los procesos de atención sobre su cuerpo, su hijo y su proceso de parto. Esto contribuye a disminuir los niveles de ansiedad, a tener un rol activo, a construir relaciones más equitativas con el personal de salud y a atender efectivamente a sus solicitudes.

No obstante, se halló que las mujeres no reciben información suficiente sobre el proceso de trabajo de parto y el parto durante los controles prenatales, el personal médico no dispone de tiempo necesario para brindar dicha educación, además que la oferta de cursos de preparación para gestantes es escasa o nula. "No me dijeron como pujar, nada, ni mucho menos, yo llegue a la clínica y fue totalmente diferente, tras de que yo pedía colaboración, que me ayudaran para saber cómo pujar para que no me doliera pero pues me dijeronahi usted debe saber, en los controles me imagino que allá le explicaron." (Informante cultural 8).

Se evidencia que la responsabilidad de brindar educación sobre el proceso de parto y parto recae sobre el primer nivel de atención, pero en las instituciones donde las gestantes realizan su control prenatal, no se generan las estrategias adecuadas para que los procesos educativos sean efectivos, la consulta de enfermería para gestantes ha casi desaparecido y el tiempo de la consulta médica es limitado.

La posibilidad de que la mujer pueda autónomamente decidir en qué posición parir es impensable, pues los protocolos institucionales consideran únicamente la posición de litotomía para la atención del parto, aun cuando existe evidencia científica que favorece la adopción de otras posiciones ${ }^{18-19}$.

Belli ${ }^{20}$ señala que se consideran actos constitutivos de violencia obstétrica obligar a la mujer a parir en posición supina y con las piernas levantadas, existiendo los medios necesarios para la realización del parto vertical. Los siguientes relatos evidencian la imposibilidad de decidir autónomamente por una posición para parir: "Yo dije que si uno se ponía en forma de cuatro el dolor es menos, y él bebe puede nacer fácil, pero no, eso fue solamente acostada y eso no me dejaron elegir" (Informante cultural 8). Otra refiere: "No, ni me dejaban hablar (el personal de salud), entré y me ubicaron; eso no me dijeron nada, me pasaron de una camilla a otra con una pierna acá y la otra allá y puje." (Informante cultural 9). 
La violencia verbal y el impedimento de las gestantes para expresarse libremente, se constituyó también en otra subcategoría a través de la cual se manifestó la violencia psicológica, que compromete el bien-estar de las mujeres que viven estas experiencias donde resulta primordial recibir apoyo. Burgo ${ }^{21}$, afirma que son fundamentales y placenteros para el desarrollo del parto: los movimientos, voces, sonidos, palabras y miradas, puesto que se convierten en la posibilidad de comunicar lo que se siente.

Sin embargo, en este estudio, las informantes claves manifestaron haber sido limitadas a expresar libremente sus emociones, sentimientos e inquietudes, optando por una actitud de sumisión, debido a regaños, críticas y burlas por parte del personal de salud. Una de las participantes manifestó que hay frases que nunca le deberían decir a una mujer mientras la están atendiendo: "Que para qué abría las patas antes y acá si se ponía a gritar" (El personal de salud hacia esos comentarios a la madre) (Informante cultural 9).

Belli ${ }^{18}$, afirma que las agresiones verbales se manifiestan a través de muestras de insensibilidad ante el dolor de la mujer, manteniendo silencio a sus preguntas, a través de la infantilización, los insultos y los comentarios humillantes (5). Una de las participantes la vivió así: "Otras dos mamitas gritaban (...) yo no gritaba y ellas sí, a una muchacha le dijeron usted pensaba que esto era como tirar una pepa de guama, así no, así no son las cosas le dijo, usted viene acá y tiene que esperar, así aprende a valorar a su mamá! - le decían porque ella gritaba- y apenas va en dos que va hacer cuando este en diez." (Informante cultural 6).

La violencia verbal en el marco de los procesos de atención obstétrica, surge entre otras razones por lo que Foucault llama "el poder/saber", el cual fue analizado por Arguedas $^{11}$, desde el campo de la obstetricia considerando que es el ejercido por parte de los profesionales en salud (especialistas en medicina y enfermería), las mujeres embarazadas o en proceso de parir no son interlocutoras válidas, ya que no hablan el lenguaje de la ciencia, por ello, no ocupan un lugar igualitario en la relación establecida dentro de la institución médica.

Arguedas ${ }^{11}$ citado por Restrepo, et $\mathrm{a}^{22}$, considera que en principio, no existe una intención de establecer un diálogo, sino más bien una relación de poder ${ }^{22}$. Esta posición desigual para la construcción de una comunicación efectiva, fue relatada así: "Uno allá no puede decir nada porque si uno se queja le dicen que uno no sabe. ¡Nosotros somos los médicos, que nosotros somos lo que sabemos! Entonces son cosas que uno tiene que aguantar y estarse callado hasta que ya. Pedirle a Dios que lo saque adelante con su hijo." (Informante cultural 1).

Generar culpa es una táctica que en el marco de los procesos de atención puede resultar efectiva para el personal de salud y que hace recordar las palabras de Freud cuando manifestó "la culpa es de la madre". "Este sentido común ha sido interiorizado hasta lo más profundo de nuestro ser - todo cuanto le pasa a un individuo es susceptible de ser responsabilidad directa de su madre" . Al respecto una informante que comparte sala de partos y presencia el trato del personal de salud hacia otra gestante manifiesta: "El bebé ya le estaba naciendo en ese momento no la llevaron a la sala de partos porque estaba ocupada, ya le estaba saliendo la cabeza y ella volvía y lo metía, la doctora decía: "-jmamá puje, mamá puje! y ella no podía. La doctora le dijo: - “iAh!, jentonces que se muera! Porque usted no quiere ayudar" y esa muchacha decía: - “Doctora, doctora, mi hijo, mi hijo, se va a morir?- ella decía: - "pero es que usted no se ayuda! - ipuje!"”(Informante cultural 2).

\section{Violencia Simbólica}

Es la que se ejerce como resultado de la dominación. Resulta del consentimiento por interiorización y/o naturalización de las relaciones jerárquicas y la normalización de este tipo de relación. Por ello existe una suerte de aceptación de las propias parturientas en tanto se ha normalizado una posición dependiente del sistema del conocimiento autorizado ${ }^{23}$.

Este tipo de violencia dificulta incluso la misma identificación de la violencia obstétrica porque al ser tan frecuente las gestantes consideran que la misma hace parte del proceso de atención al que deberán "someterse". Una de las participantes señaló "porque allá todo el mundo es igual entonces así uno le diga a quien le diga no hacen algo diferente, entonces para que uno hacerse coger rabia" (Gestante que no manifestó a otra persona la incomodidad de haberla dejado sola y el recibir un trato diferente) (Informante cultural 7).

El sentimiento de culpa aflora con frecuencia cuando las mujeres resultan víctimas de esta modalidad de violencia: "Yo siento que hice un mal trabajo (trabajo de parto), porque hubo una enfermera que me mató la moral, porque me dijo - "así como lo hizo, ahora puje, pero que no grite, que de nada le sirve"-, y yo me senti muy mal sin ganas ni nada" (Informante cultural 8). 
La violencia simbólica, se traduce en una actitud de sumisión, adquirida por las mujeres como medio de protección ante una inadecuada atención, como regaños o aislamiento. De igual forma, la docilidad por parte de las gestantes resulta de la naturalización de la violencia en los procesos de trabajo de parto y parto, por interiorizaciones resultantes de experiencias anteriores, por eventos que presenciaron en la atención o por relatos de otras mujeres.

\section{Violencia sexual}

Como un resultado emergente o muy poco documentado dentro de las investigaciones sobre violencia obstétrica, resultó preocupante e indignante encontrar un caso de violencia sexual que se determina como cualquier tipo de comportamiento que busca la coacción hacia una persona para que lleve a cabo una conducta sexual especifica. Entendiéndose en la presente investigación como insinuaciones o comentarios sexuales en medio del desarrollo del proceso obstétrico y que fue categorizada como "Dolo en el acto médico".

La Organización Mundial de la Salud (OMS) ${ }^{24}$ define la violencia sexual como: "todo acto sexual, la tentativa de consumar un acto sexual, los comentarios o insinuaciones sexuales no deseados, o las acciones para comercializar o utilizar de cualquier otro modo la sexualidad de una persona mediante coacción por otra persona, independientemente de la relación de ésta con la víctima, en cualquier ámbito, incluidos el hogar y el lugar de trabajo"24.

De acuerdo con esta investigación, este tipo de actos también están presentes en los escenarios de atención del parto, una informante cultural manifestó insinuaciones sexuales por parte del médico mientras le realizaba los tactos vaginales: "Lo que no me gustó fue que un ginecólogo se sentó a mi lado y me dijo que si a la hora en el que él me tocaba en el tacto yo sentía placer. Eso me incomodó mucho y me dio miedo". Esta gestante manifestó que sintió mucho miedo durante el tiempo que duró la atención porque el médico estuvo de turno durante todo este periodo.

Es claro que ante la negación de tener acompañante durante la atención, no existe la posibilidad ni siquiera de demandar un acto abusivo de esta naturaleza pues no hay testigos. Es importante recalcar, que la mujer en estado de gestación se ve obligada a exponer su cuerpo $\mathrm{y}$ en especial sus órganos genitales, generando una sensación de mayor vulnerabilidad al ser atendida por el personal masculino.
A pesar que este relato es el único en el que se evidenció este tipo de violencia, no significa que hechos como estos no ocurran. Muestra una especial gravedad por presentarse durante los procesos de atención del trabajo de parto y parto al interior de las instituciones hospitalarias, degradando el acto médico y constituyendo una flagrante violación a los derechos humanos de las mujeres. Muestra también una completa actitud de sumisión y de incapacidad de las mujeres para enfrentar este tipo de violencia y el desconocimiento de sus derechos.

Esta investigación evidencia una alta frecuencia de la violencia obstétrica en donde sus diferentes modalidades se sobreponen de una forma $u$ otra en una misma experiencia, dando cuenta del drama que viven las mujeres ante la violación de sus derechos humanos en un contexto donde deberían sentirse protegidas. Sin embargo, se hace necesario destacar los esfuerzos de muchos miembros del equipo de salud y sus instituciones que logran brindar una atención humanizada y de calidad, representada para esta investigación en 31\% de los casos de mujeres que no vivieron experiencias de violencia obstétrica.

A su vez, los miembros del equipo de salud, terminan en muchos casos ejerciendo violencia obstétrica aún sin plena conciencia de lo que sus acciones u omisiones pueden representar en la vida de las mujeres que atienden, pues el peso del modelo médico hegemónico, el mantenimiento de patrones culturales machistas, el cumplimiento de los protocolos de atención y de las políticas institucionales, las responsabilidades y sobrecarga laboral que se les asigna desde el sistema de salud, así como una infraestructura que no garantiza la privacidad y el acompañamiento de un familiar significativo; permean todos los procesos de atención y comprometen seriamente la posibilidad de garantizar un parto y nacimiento respetuoso, protegido y con-sentido.

\section{Recomendaciones para la acción}

Las acciones para garantizar una atención respetuosa del parto y el nacimiento, deben enmarcarse desde la garantía de los derechos humanos de las mujeres y por tanto a las autoridades, las Empresas Promotoras de Salud, las universidades, las asociaciones de usuarias/os, las organizaciones sociales y de mujeres, les corresponde asumir el compromiso de liderar los esfuerzos institucionales y comunitarios para que las mujeres, sus parejas y familias puedan vivir esta experiencia vital con un cuidado humanizado, cálido, respetuoso y seguro, brindando una información 
completa y oportuna que le permita a la mujer tener autonomía sobre las decisiones que involucren su salud y la de su hijo y que responda a las necesidades de atención integral de calidad, desde las dimensiones físicas, emocionales, culturales y espirituales.

Se hace necesario además velar por el cumplimiento de derechos como: ser escuchada, saber acerca del tratamiento y los procedimientos, mantener la confidencialidad y respetar sus creencias, costumbres y opiniones, es decir que dentro de las instituciones se visualice a las mujeres como ciudadanas sujetas de derecho y no solo usuarias receptoras de servicios ${ }^{25}$.

Para dar cumplimiento a estas actividades se recomienda que el personal de salud que acompañe el trabajo de parto y el parto, vele por el cumplimiento de los derechos de los pacientes, enfocándose en el respeto, la tolerancia y la contención. Además, garantizar la protección de su intimidad y el consentimiento para la realización de los procedimientos aún aquellos que son considerados de rutina.

Permitirle a la mujer expresar sus emociones y sentimientos, elegir la posición para parir y mantener una comunicación eficaz que garantice la apropiación de su proceso de parto, favorece el empoderamiento de la gestante, dándole control sobre su cuerpo, aumentando el grado de satisfacción y facilitando el trabajo de parto.

Se hace necesario promover en los equipos de salud y en las familias el ejercicio de una paternidad activa. Desde el rol de enfermería se pueden favorecer acciones que involucren la participación del padre desde la atención prenatal, de tal manera que el acompañamiento del padre en el trabajo de parto y parto sea efectivo, favoreciendo el vínculo padre - hijo, las relaciones de pareja y una participación activa del padre en los procesos de crianza. La educación y consulta de enfermería durante el control prenatal es indispensable para mejorar el conocimiento de las mujeres sobre el proceso del parto, de tal manera que la gestante tenga un rol más activo y autónomo durante la atención.

Los cambios en la infraestructura pueden también favorecer la creación de espacios más cómodos, íntimos y cálidos en donde la presencia del padre no interfiera con la atención a otras gestantes, de tal manera que los espacios favorezcan que la mujer recupere la autonomía sobre el ritmo del proceso del trabajo de parto con la ayuda de artefactos de bajo costo que pueden ser incorporados en estos espacios como hamacas, pelotas, colchonetas, pebeteros con esencias florales, música, entre otros, de tal forma que el rol del personal de salud sea de acompañante en la etapa final de una larga espera que se constituye en el inicio de la aventura de convertirse en padre y madre a través de una experiencia vivida gozosamente mientras se cuida la salud y las experiencias vitales de madre - recién nacido - padre.

\section{Conclusiones}

Aunque la violencia obstétrica puede presentarse en cualquier tipo de mujer sin importar su condición socioeconómica o educativa, la mayoría de entrevistadas tenían un nivel educativo bajo, condición supone un nivel de empoderamiento también bajo que contribuya a la exigencia de derechos.

De las diferentes modalidades de violencia obstétrica, en la investigación se evidenciaron todas las variaciones de este tipo de violencia, predominando la psicológica e institucional; sin embargo, uno de los hallazgos más alarmantes en la investigación, es el caso de la violencia sexual en la atención obstétrica. Frente a estas situaciones, la mayoría de mujeres no denuncia, pues desconocen sus derechos o no están en condiciones de exigirlos y/o naturalizan los actos de violencia obstétrica.

Los resultados de esta investigación son un llamado a la acción a las entidades de gobierno, a las instituciones de salud, a la academia y a las organizaciones sociales y de mujeres, para eliminar cualquier tipo de violencia en el proceso de atención del trabajo de parto y parto, para construir un diálogo horizontal entre la gestante $\mathrm{y}$ el personal de salud y favorecer el ejercicio de la ciudadanía reproductiva en la prestación de los servicios.

El personal de salud debe reconocer en su formación las distintas modalidades de violencia obstétrica con el fin de: diseñar mecanismos para su prevención, sanción y abolición; aportar a la construcción de un marco jurídico que castigue ejemplarmente toda forma de violencia obstétrica, tal como existe en México, Venezuela y Argentina; divulgar los derechos de las gestante, su autoconocimiento y su autoconfianza entorno a las dinámicas fisiológicas, emocionales y espirituales en el trabajo de parto, parto y puerperio y promover la paternidad activa.

Corresponde a los profesionales de enfermería liderar iniciativas que permitan a las gestantes, escoger libremente la persona que acompaña el trabajo de 
parto y parto y la forma de parir, además de garantizar el respeto por la "hora sagrada" (contacto piel a piel en la primera hora de vida) y el arrastre al pecho. Recientemente el Ministerio de Salud y Protección Social emitió la circular 000016 de 2017, la cual busca el fortalecimiento de acciones que garanticen la atención segura, digna y adecuada de las maternas en las instituciones de salud a lo largo de todo el territorio Nacional, se espera que su aplicación contribuya a eliminar esta forma de violencia contra las mujeres y sus hijos por nacer.

\section{Agradecimientos}

A todas las mujeres que desde su experiencia de la maternidad aceptaron a participar de este proceso con la esperanza de que sus testimonios puedan contribuir a un parto y nacimiento respetado para las futuras generaciones.

\section{Consideraciones éticas}

Esta investigación se basó en los principios estipulados en CIOMS/82 -2002 y en la resolución 008430 de 4 de octubre de 1993, en la que se establece las conductas científicas, técnicas y administrativas para la investigación en salud, TITULO II: de la Investigación en Seres Humanos, Capítulo I: de los Aspectos Éticos de la Investigación en Seres Humanos: en toda investigación en el que ser humano sea objeto de estudio, deberá prevalecer el criterio del respeto a la dignidad y la protección de los derechos y bienestar, y en conjunto con el Artículo 11, la investigación se consideró sin riesgo, ya que no se realizó ninguna intervención o modificación intencionada de las variables biológicas, fisiológicas, sicológicas o sociales de los individuos.

\section{Conflicto de intereses}

No existe conflicto de intereses en el trabajo realizado.

\section{Referencias}

1. Bruyn M. La violencia, el embarazo y el aborto. Cuestiones de derechos de la mujer y de salud pública. Un Estudio de los datos mundiales y recomendaciones para la acción. Segunda edición. Chapel Hill, EE.UU: 2003; 5.

2. Minsalud.gov.co. Violencias de Género. Bogotá; 2016.

3. Organización Mundial de la Salud - OMS. Ginebra, Suiza; 2014. Prevención y erradicación de la falta de respeto y el maltrato durante la atención del parto en centros de salud.

4. Organización Mundial de la Salud - OMS. Ginebra, Suiza; 2015. Epidemia de cesarías innecesarias en América Latina.

5. Hernández-Rojas C, Vera Quintal A. Violencia obstétrica en La Gran Caracas. Estudio multicéntrico, Venezuela. Trabajo de grado. Especialista en Obstetricia y Ginecología. Caracas. Universidad Central de Venezuela; 2012. 63 p.

6. Villanueva-Egan L.A. El maltrato en las salas de parto: reflexiones de un gineco-obstetra. Rev CONAMED. 2010; 15(3): 147-151.

7. Grupo de Información en Reproducción Elegida. Violencia obstétrica. Un enfoque de derechos humanos. México: c2015. 104 p.

8. Quevedo P. Violencia Obstétrica: una manifestación oculta de la Desigualdad de Género. Argentina. [Tesis Trabajo Social] Universidad Nacional del Cuyo. 2012; 314

9. Vallana-Sala VV. Parirás con dolor, lo embarazoso de la práctica obstétrica Discursos y prácticas que naturalizan la violencia obstétrica en Bogotá. Tesis maestría en Estudios Culturales. Bogotá: Universidad Javeriana; 2016. 143 p.

10. Ministerio de Salud y Protección Social. Colciencias. Guía de práctica clínica para la prevención, detección temprana y tratamiento del embarazo, parto o puerperio. Bogotá; 2013. 84 p.

11. Arguedas G. La violencia obstétrica: propuesta conceptual a partir de la experiencia costarricense. Cuad Intercamb Centroamérica Caribe. 2014; 11(1): 145-169. doi: 10.15517/C.A..V11I1.14238.

12. Villalón H, Toro R, Riesco I, Pinto M, Silva C. Participación paterna en la experiencia del parto. Rev Chil Pediatr. 2014; 85(5): 554-560. doi: 10.4067/S0370-41062014000500005.

13. Aguayo F, Correa P, Kimelman E. Estudio sobre la participación de los padres en el sistema público de salud de Chile. Informe final. Santiogo de Chile; 2012. $160 \mathrm{p}$.

14. Organización Mundial de la salud - OMS. [Internet]. Ginebra, Suiza. Recomendaciones de la OMS para los cuidados durante el parto, para una experiencia de parto positiva Transformar la atención a mujeres y neonatos para mejorar su salud y bienestar. 2018.

15. Villanueva Egan L.A. El maltrato en las salas de parto: reflexiones de un gineco-obstetra. Rev CONAMED. 2010; 15(3): 148-151.

16. CN. cimacnoticias. México D.F. Sororidad nuevas prácticas entre las mujeres; 2014 .

17. Davis-Floyd, Robby. Feminist theory in the study of folklore. Illinois: 1993. p. 297-326. 
18. Sampaio A, de Vasconcelos A, Ferreira E. Atención humanizada del parto de adolecentes: ¿norma o realidad? Rev Chil Obstet Ginecol. 2008.. http:// dx.doi.org/10.4067/S0717-75262008000300008.

19. Organización Mundial de la salud - OMS. Ginebra, Suiza. Recomendaciones de la OMS para la conducción del trabajo de parto. 2015.

20. Belli L.F. La violencia obstétrica: otra forma de violación a los derechos humanos. UNESCO. Rev Red Bioética. 2013; 1(7): 25-34.

21. Burgo C. Parto humanizado, Buenos Aires. Los derechos de la embarazada.

22. Restrepo Sanchez A, Rodriguez Martinez D, Torres Castro NM. Me des-cuidaron el parto: la violencia obstétrica y el cuidado recibido por el personal de la salud a mujeres durante su proceso de parto. [Trabajo de Grado Enfermería] Pontificia Universidad Javeriana. 2016. $170 \mathrm{p}$.

23. Peña Collazos W. La Violencia Simbólica como reproducción biopolítica del poder. Rev Latinoam Bioética. 2009; 9(2): 62-75. doi: https://doi. org/10.18359/rlbi.1060.

24. Organización Mundial de la salud - OMS. [Internet]. Ginebra, Suiza. Comprender y abordar la violencia contra las mujeres; 2019.

25. Valdez Santiago R, Salazar Altamirano Y, Rojas Carmona A, Arenas Monreal L. El abuso contra las mujeres durante la atención del parto en hospitales públicos en México. Rev CONAMED. 2016; (suplemento 1): 42-47. 